JSCM T

Journal of Sustainable Construction

Materials and Technologies
Journal of Sustainable

Construction Materials

and Technologies

J Sustain. Construct. Mater. Technol. 1(1) (2016) 16-22

www.eds.yildiz.edu.tr/jscmt

\title{
Cleanliness of ventilation ducts, indoor space and AHU
}

\author{
Aleš Rubina ${ }^{1, \text { a, *, Olga Rubinováa }}{ }^{2, b}$ and Petr Blasinski ${ }^{3, c}$ \\ ${ }^{1,2,3}$ Department of building services, Brno University of technology, Faculty of civil engineering, \\ Czech Republic \\ arubina.a@fce.vutbr.cz, ${ }^{b}$ rubinova.o@fce.vutbr.cz, ${ }^{c}$ blasinski.p@ fce.vutbr.cz
}

Received January 8, 2016, accepted February 28, 2016

\begin{abstract}
Paper deals with analysis of cleanliness air conditioning duct for various stages of construction. There were taken samples and these were analyzed for different type of storage duct on construction. They are compared differences between the "right" stored duct and unprotected duct including the actual interior space and air handling units serving. In the conclusion of this article is summarized the impact of carryover dirt from the duct and an air handling unit to the ventilation system.
\end{abstract}

Keywords: duct, contamination, cleanliness of cleaning spaces, air handling units;

\section{Introduction}

Air handling systems comprise a device for air transport and treatment (HVAC unit), sub-elements for the regulation, reduction of sound, and in particular the distribution pipe system. The authors with the support of the specific research project FAST-S-15-2620 are currently carrying out data collection and evaluating the cleanliness of air pipe distribution at several stages of their life cycle. In the article, the authors focus mainly on pollution of air ducts with solid aerosols - dust. As follows from the previous measurements and investigations, crucial phase for the contamination of pipe ducts is the implementation phase of construction and installation.

\subsection{Cleanliness of ventilation ducts}

Installation of piping of HVAC equipment is carried out mostly at the same time, regarding in terms of time requirements and deadlines for construction activities. Realization and construction of another rough or finishing construction works are following after installing ductwork. At this stage discipline of persons involved in the installation of air conditioning is absolutely important. As shown in the Fig. 1, building in stage of installation of piping and inconsistency protect of piping from their inner sides may cause, and also causes, substantial contamination.

From Fig. 1 can be seen that the contamination inside the pipe occurred before placing or connecting other pipes. The result is a practical destruction of pipework not only of the contaminated part, but then the whole duct route airflow. That the contamination is happening regularly, samples from many other buildings confirmed, see Fig. 2. The mistake is the inconsistent protection of pipes in the time between assembling the arches and straight pipes, after arches installation the airway wasn't blend.

To be able to quantify the contamination and undertake corrective measures, it is necessary to act in accordance with valid or obligatory regulation. DIN EN 15780 [1] is the basic regulation, which quantifies the quality of cleanliness of HVAC systems. According to this standard there are defined three basic acceptable

\footnotetext{
${ }^{*}$ Corresponding author. Aleš Rubina

E-mail address: rubina.a@fce.vutbr.cz 
classes of cleanliness. At handover to the user clean rooms HVAC should conform to the requirements of graded acceptable cleanliness class $\mathrm{C}$ - High cleanliness class.
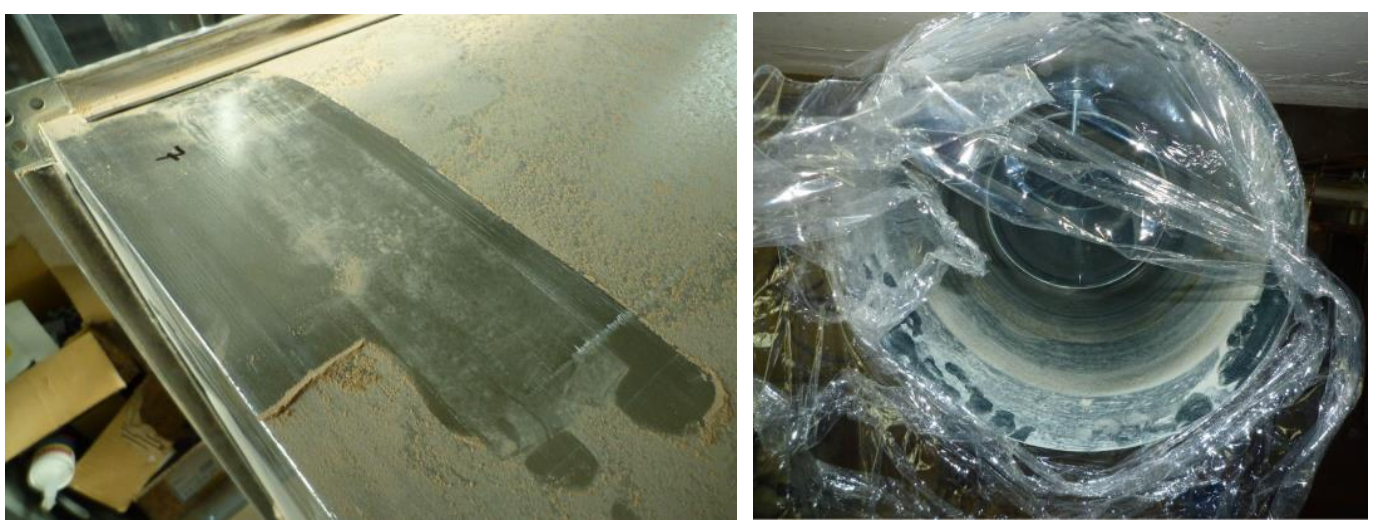

Figure 1. Sample contamination during ducts assembly (on the outside - left, on the inside - right)
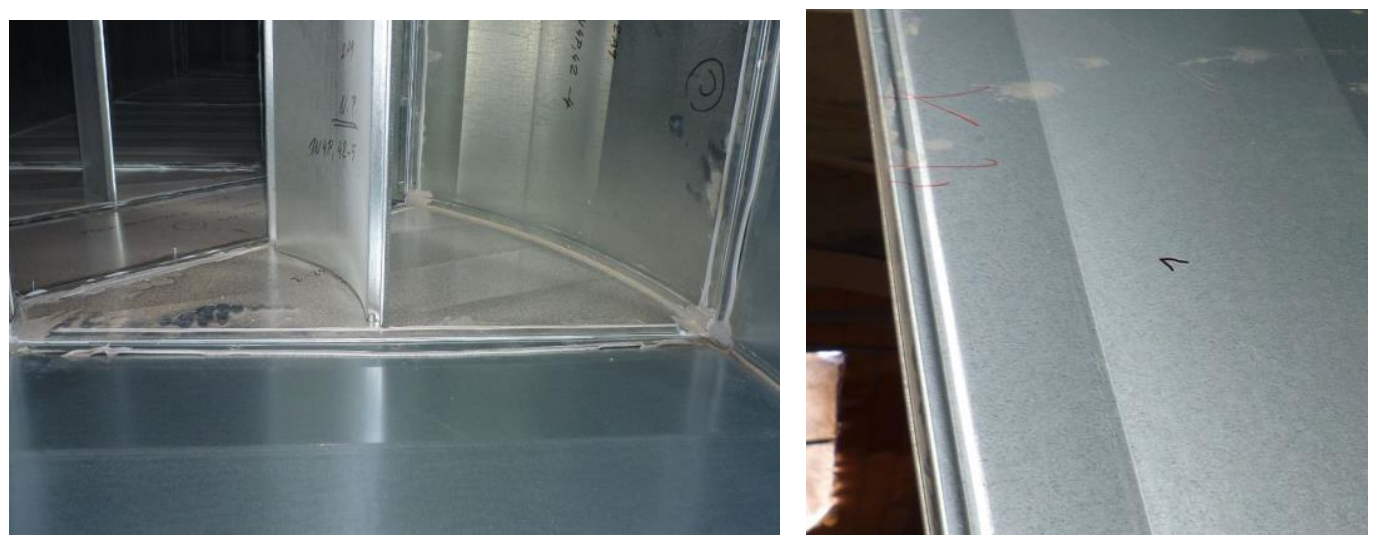

Figure 2. Sample of duct contamination in response to poor protection duct in time between two consecutive sections (left), the cleanliness of the air duct before assembly (right).

\subsection{Classification of acceptable cleanliness classes:}

A low $\quad-\quad$ rooms without permanent presence of persons, e. g. warehouses, technical rooms.

B medium _ - offices, hotels, restaurants, schools, theaters, residential buildings, shopping areas, exhibition buildings, sports buildings, common areas in hospitals and general spaces in the industry.

C high _ - laboratories, treatment rooms in hospitals, representative offices, air handling units in hygienic design.

The acceptable level of dust deposits in this case defines the acceptable level of cleanliness of the new air duct at the handover from the contractor to the user. In practice, this corresponds to the contamination which is considered as acceptable. Quantification of dust deposit is shown in table 1.

Table 1. Definition or quantification of contamination of the airway clogged by dust [1].

\begin{tabular}{ccc}
\hline $\begin{array}{c}\text { Cleanliness } \\
\text { class }\end{array}$ & $\begin{array}{c}\text { The acceptable level of dust } \\
\text { deposits } \\
\text { Supply, circulating air duct }\end{array}$ & $\begin{array}{c}\text { The acceptable level of dust } \\
\text { deposits } \\
\text { exhaust air duct }\end{array}$ \\
\hline Low & $<0,9 \mathrm{~g} / \mathrm{m}^{2}$ & $<1,8 \mathrm{~g} / \mathrm{m}^{2}$ \\
Medium & $<0,6 \mathrm{~g} / \mathrm{m}^{2}$ & $<1,8 \mathrm{~g} / \mathrm{m}^{2}$ \\
High & $<0,3 \mathrm{~g} / \mathrm{m}^{2}$ & $<0,9 \mathrm{~g} / \mathrm{m}^{2}$ \\
\hline
\end{tabular}

To fulfil the required classes of cleanliness the samples should exhibit lower final values than the limit values. After cleaning the pipes and other relevant parts the level of contamination after cleaning should be less than $0.3 \mathrm{~g} / \mathrm{m}^{2}$. Cleanliness or contamination is primarily evaluated visually, which can be confirmed by 
measurement. After assembling or before starting the air ducts must be first inspected visually, both in assessing the need for cleaning and verification of cleaning results. Objective measurements are used in the case that the results of visual inspections in terms of cleanliness and cleaning necessary are in mutual inconsistency.

For visual inspection it is possible to use prepared precise benchmarks of contamination and compare them against the collected samples. However, for objective evaluation it is necessary to weigh the samples taken from a well-defined area on the precise scales. Due to the complex logistics of sampling on site, error measurement on precise scales (moisture of aerosol, etc.) and the overall complexity of the process of objective evaluation there is on the Technical Building Services Institute of Brno University of Technology, Faculty of Civil Engineering, developed the methodology of microscopic assessment of the contamination of air ducts by fixed aerosol. This method is fast and enables trouble-free feasibility of assessment directly in the realization on the construction site. Practically, it is a more accurate standard visual inspection with the physical benchmark, when the data will be electronically drawn off and compared with the electronically defined benchmark (continuously replenished databases). The sample of electronic measuring by the microscope shows the Fig. 3.
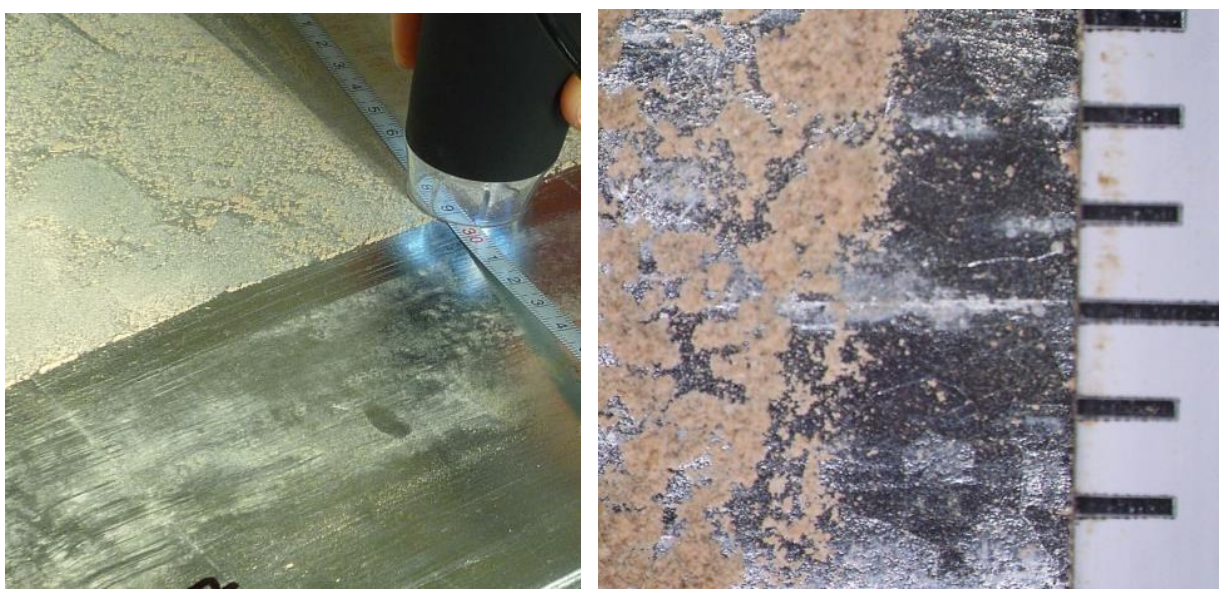

Figure 3. Example of sampling by the microscope (left) and the result (right)

On the Fig. 3 we can observe quantity of construction dust witch is compared to the investigated surface of the pipe portion - texture of pure pipe formed from galvanized sheet (dark and bright crystals). To illustrate the real size of the dust particles there is in the right part of the figure enclosed scale with the size of $1 \mathrm{~mm}$. The result of such high contamination is evident. The cleanness of the air duct is considered much higher than the permissible value of low grade. With the higher resolution it is then possible to perform the calculation or assessment of the amount of dust grain and then to define the level of contamination.

To be able to apply the methodology of dust particles microscopy, it is necessary to confront it and validate it according to the certified method. Within the specific research there are carried out at the same places sampling for visual comparison of contamination with standard sample (i.e. benchmark). Other examples of the sampling spot are shown in Fig. 4.
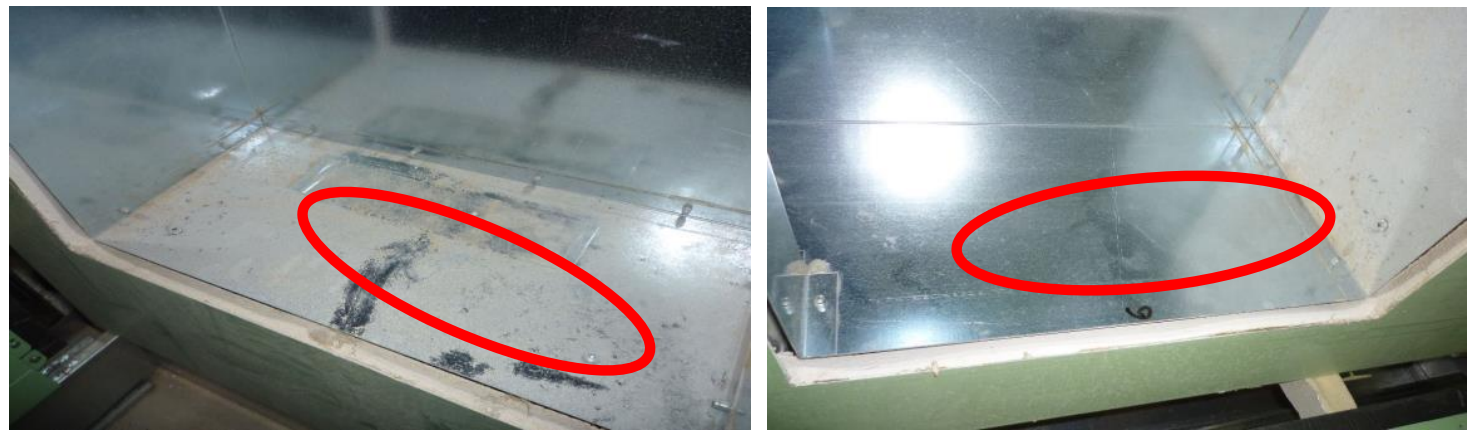

Figure 4. Demonstration of sampling by using tape for comparison with a standard dust concentration

As apparent from Fig. 5 according to the degree of contamination, the taken sample can be compared with benchmark and subsequently it is possible to define the degree of contamination in $\mathrm{g} / \mathrm{m}^{2}$ of considered area.

For the successful result of the purity of the reference indoor environment it is therefore necessary to have a comprehensive approach to cleanliness as a complex. Currently, in particular clean rooms are built as internals in the enclosed space of the building. The built-in space consists mostly of sandwich side panels and a tight 
ceiling with fitted lights. All technical installation, pipe and wiring devices are installed above the false ceiling construction and in the shell of side panels. The built-in clean room example is shown in Fig. 6.
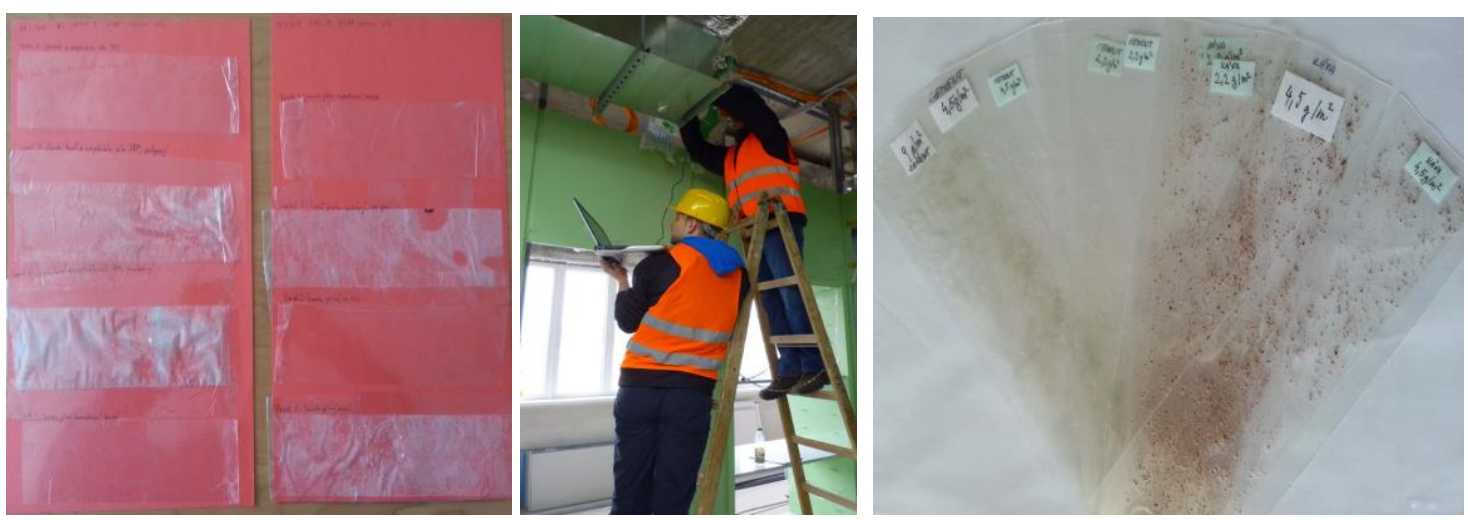

Figure 5. Example of assessment of pipework contamination by visual inspection using checking benchmark method (right), the samples (left)

\subsection{Cleanliness of indoor space}

The task of building structures of these built-in parts is to ensure airtight envelope of clean room and its cleanability, among other things. In practice, we meet with regard to the terms of realizations and implementation of various subcontracting division into smaller units with the problem of the continuity of individual technical professions and lack of time to properly clean the installation and cleaning around the builtin surrounding. On pipe installations in ceilings occur large amounts of dust, rubble and various mounting unused portions of the connecting and assembling material. Especially fine particles sized from 0.1 to 1.0 microns, with regard to their own weight, they do not settle and are thanks to different air streams transported from the surroundings to the clean built-in spaces. Pressure conditions in the built-in space, which of course varies during realization (overpressure, underpressure, opening doors, etc.), have a considerable influence on the transport of these particles from surrounding to the built-in inner space. Another factor, which in turn affects the inner cleanliness and continuous contamination of the interior space, is a lack of discipline of staff involved in its construction.
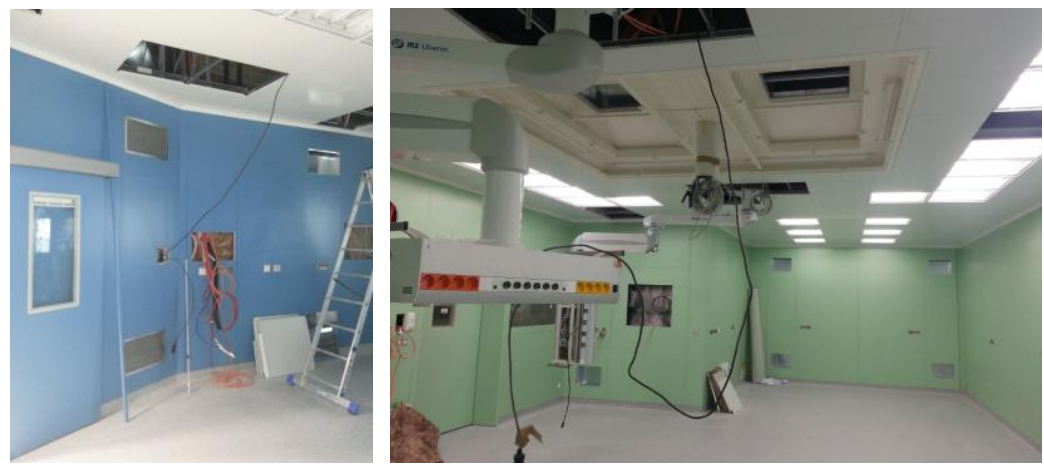

Figure 6. Demonstration of technical and design solution of bulit-in cleanrooms

Fig. 7 is a sample of a multi-stage cleaning the operating room before putting it into commissioning. Cleaning is divided into several phases. Figure 7 presents the final stage of the so-called inner space disinfection. As shown by experiments, in principle it is necessary to carry out purification in the following steps:

- dry mechanical cleaning to remove larger particles of solid matter, at least 1 time (all structures surfaces)

- wet cleaning to remove fine particles, grease etc., at least 1 time (all surfaces of structures including lights, surfaces of technology etc.)

- wet chemical cleaning to eliminate not only solids, but also living organisms, so-called space disinfection, at least 3 times (all surfaces of structures including lights, surfaces of technology etc.) 

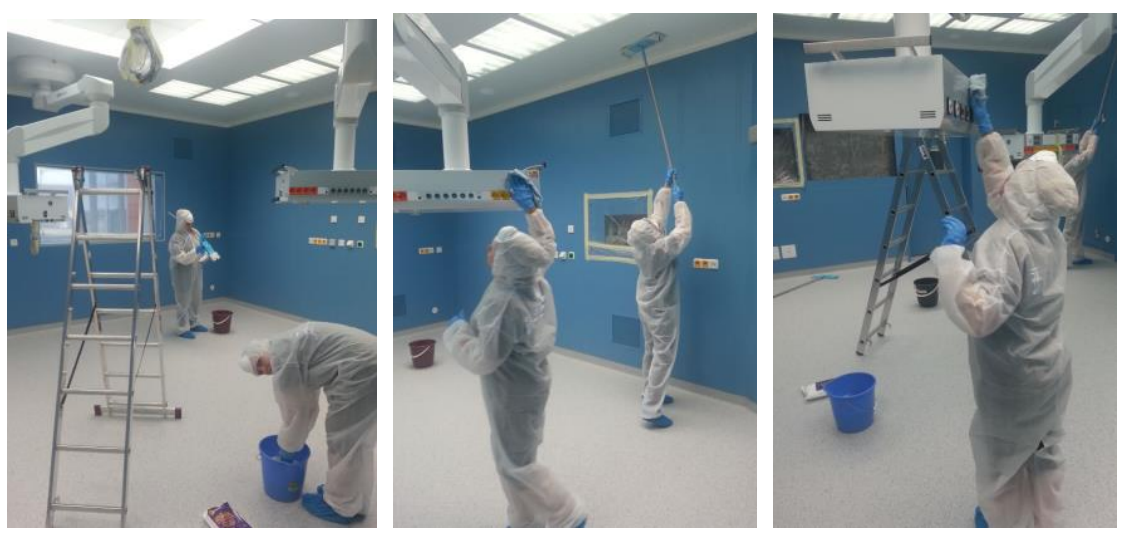

Figure 7. Demonstration of the final phase of the clean room disinfection

As demonstrated by measurement and experimentation in the implementation of clean rooms of various buildings, after several times of cleaning dust contamination of the interior construction occurs due to a leakage and it takes a long time before the construction as a whole becomes so-called clean. In combination with the dirty air ducts and thus installed HEPA filters the degradation of the cleanliness class of the reference space occurs in the first few months of operation.

For example, when cleaning the space shown in Fig. 7, it becomes contaminated by very fine solid aerosol after three cycles of said wet cleaning stages in the time between these phases, which takes about 20-24 hours. Physically, these particles can be seen in the cleaning area formed of special mop Fig. 8.
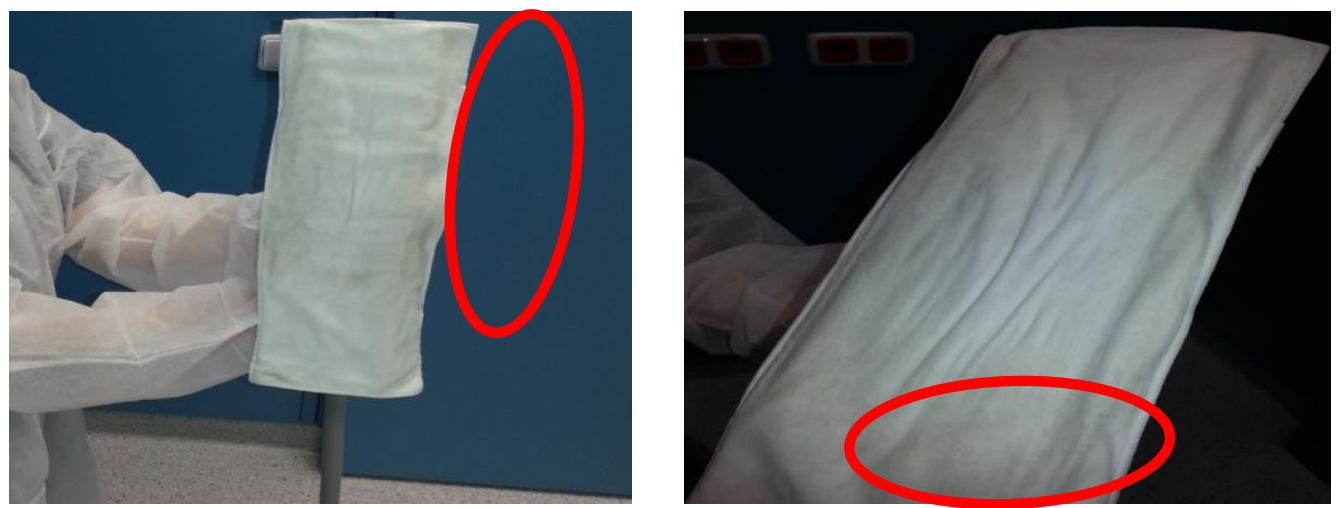

Figure 8. Sample contamination of mop head during the fourth cycle of wet cleaning phase

\subsection{Cleanliness of air handling unite}

Similarly to the inner surface of the built-in space it must be treated air conditioning system as well. Even if it is not a clean interior space, but the so-called standard interior spaces, it is necessary before handing over the work the user to perform a proper adjustment and cleaning. The cleaning procedure with respect to particles of solid matter (dust) can be carried out in accordance with the above mentioned Czech technical standard EN 15780

Minimum cleanliness of the air ducts and equipment for areas with a permanent presence of people is defined as medium cleanliness for clean rooms, laboratories and even e.g. representative offices. The technical standard required quality or class as high. Conventional pollution of HVAC equipment which is operating in the urban agglomeration is presented in Fig. 9. The research carried out on real HVAC equipment indicates that the equipment contaminated by construction activities are following the same pollution with the device after 12 months of operation without maintenance and cleaning.

The performed practical experiments resulted the cleaning procedure, which was verified by measuring the number of living organisms found in the air-conditioning equipment cleaned regularly. This complex process can be defined as follows:

- dismantling of used filters

- dismantling droplet eliminator

- mechanical cleaning

- wet cleaning

- chemical cleaning 
- refitting cleaned droplet eliminator

- installation of new "clean filter"
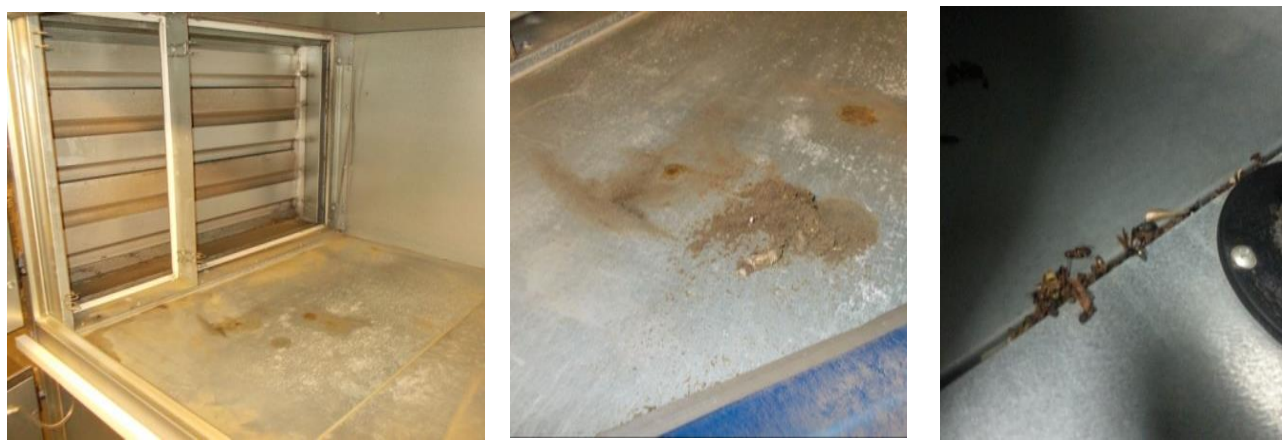

Figure 9. Demonstration of pollution of the filter chamber on the side of the intake of fresh air (left), solids trapped in the joint between the panels (right)

Rough cleaning brush, then gently clean sweep and vacuum cleaning are the most common operations used for mechanical (dry) cleaning unit according to the degree of solids clogging. For the cleaning of exchangers (cooler, heater, heat recovery, etc.) it is commonly used mechanical cleaning with compressed air. The most frequent mechanical cleaning elements are presented in Fig. 10. The rule to follow for mechanical cleaning is that the method of coarse or fine cleaning unit corresponds to the degree of pollution.
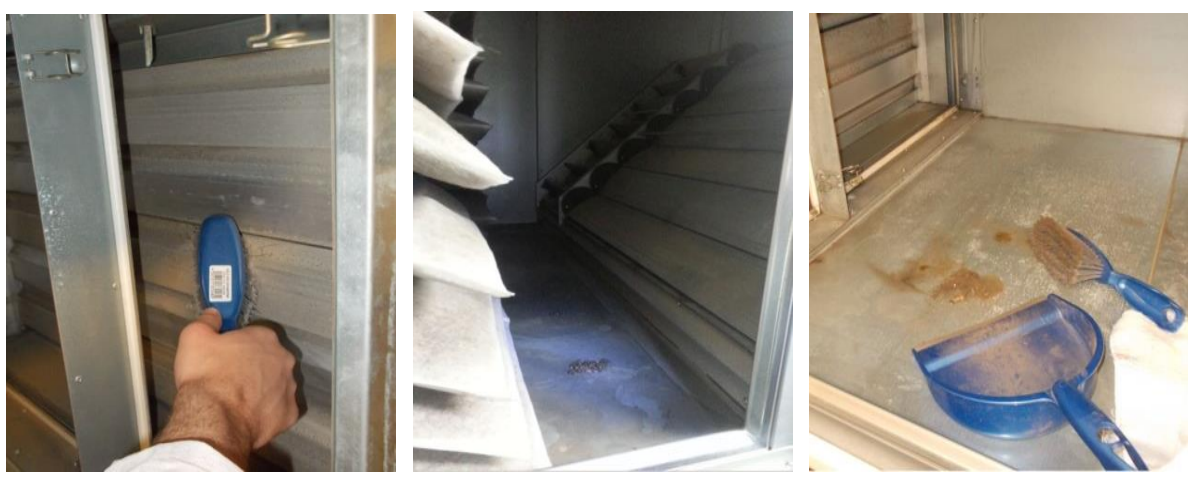

Figure 10. Sample of methods of mechanical cleaning

Wet cleaning is following after mechanical dry cleaning, and its purpose is to remove all mechanical and liquid particles that are visible to the naked eye. For example it is cleaning all surfaces of unit, frame, cladding, fans, heat exchangers, condensate tray, (very important is moist chamber), etc. Wet cleaning is done according to the degree of contamination and the type of use of air handling unit, either with clean water at room temperature, or solution of water and detergent at room temperature with subsequent necessary second washing with clean water. After wet cleaning the air handling unit must be wiped up dry.
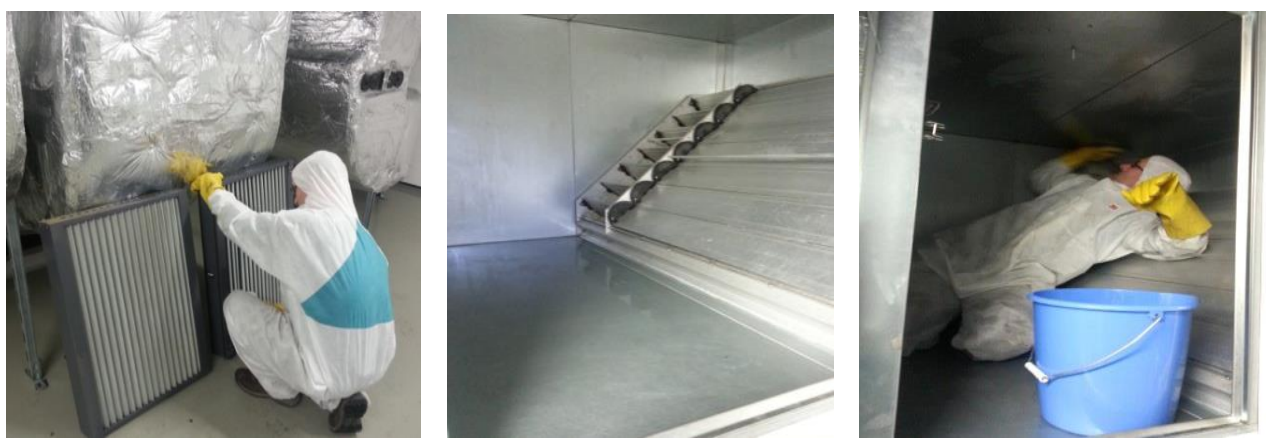

Figure 11. Sample of typical examples of wet cleaning (left droplet eliminator, right filter chamber)

The so-called chemical cleaning phase follows after the wet cleaning. The chemical is applied on the precleaned surface inside the unit. The aim is to maximum of eliminate the possible microbial structures in the interior of the unit - disinfection of the unit. Most often it is a mixture of water and disinfectant, the application performs with the mist spray solution to all parts of the unit. An important factor is not to miss enclosures, heat 
exchangers, folds etc. After application the unit is closed and disinfection is allowed to act for a predetermined time.
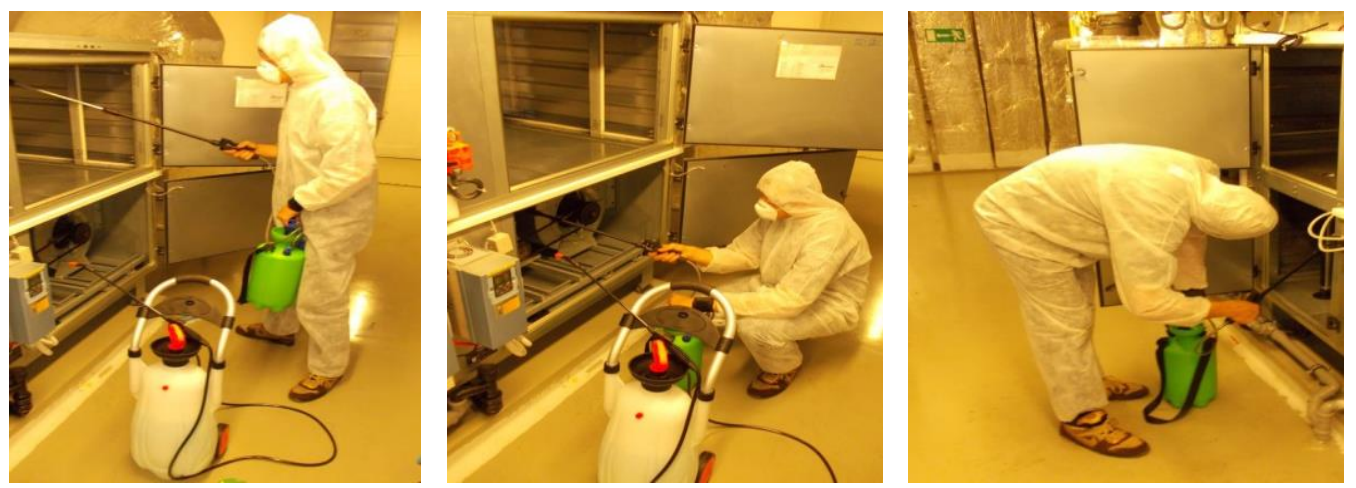

Figure 12. Sample of the dry cleaning

\section{Summary}

The aim of the article was to acquaint readers with the problem of contamination of ductwork and air handling units. The text makes it clear that at present, when buildings are constructed above standard speed and the contracted price of the work does not cover nearly overhead delivery, installation and especially the quality of installation is a very crucial factor that affects the quality of transported air and the internal microclimate. In the case of put into operation the contaminated state of device it threatens not only the continuous contamination of the inner space by emitted settled particles, but also shorten the life of the realized device.

The demonstrated methods for determining contamination and subsequent cleaning methods that the staff of Institute of Building Services utilize in practice, permit during construction effective control and propose realistic recommendations for possible correction of pollution of air ducts and in the time when it was still not too late. The result is the successful realization of high-quality results of the internal environment.

Objective measurement that is not possible due to the limited scope of this article to present here shows that regular maintenance and complex cleaning of equipment (phase prior to handing over of equipment to use and subsequent periodic maintenance) leads to a significant decrease in pollution of air-conditioning systems by solid aerosol and living organisms, (validation measured by the number of microorganisms with swabs or by an aeroscope). Regularity in this case means adherence to timetable cleanliness. The minimum interval for cleanroom is quarterly, for less demanding spaces at least half-yearly execution of complex cleaning and replacement filters.

\section{References}

1. ČSN EN 15780 - Ventilation for buildings - Ductwork - Cleanliness of ventilation systems.

2. RUBINA, A.; UHER, P., Cleaning the air handling units, article in Topenářství, ISSN 1211-0906, Technické vydavatelství Praha, spol. s r.o., Praha, 2014

3. RUBINA, A.; UHER, P.; HIRŠ, J., Methodology for the design, manufacture, installation and operation of the air handling units in hygienic design, spec. publication, ISBN 80-903586-5-9, Litera Brno, Brno, 2013

4. BJÖRKROTH, M., V. ASIKAINEN, O. SEPPÄNEN. Cleanliness criteria and test procedures for cleanliness labelling of HVAC components. In: Indoor Air 2002 The 9th International Conference on Indoor Air Quality and Climate. Monterey, California: 2001, s. 6.

5. HOLOPAINEN, R., M. TUOMAINEN, V. ASIKAINEN. The effect of cleanliness control during installation work on the amount of accumulated dust in ducts of new HVAC installations. In: Indoor Air 2002 The 9th International Conference on Indoor Air Quality and Climate. Monterey, California: 2001, s. 7.

6. ASIKAINENA, V., R. HOLOPAINENB, A. MAJANENB. The verifying concept for the cleanliness of HVAC systems. In: Lifa air [online]. 2015 [cit. 2015-06-24]. Available from: http://www.lifa.net/research/poster3_LIFA_verifying_concept.pdf 